Angeln finden sich im prähistorischen Norwegen in sehr großer Zahl und vor allem viel häufiger als Harpunen. Gewisse Felszeichnungen lassen an Leinenfischerei denken. Erwähnenswert ist wohl auch die ebenfalls alte Verwendung von Netzen, worauf steinzeitliche Garn-Nadeln zu ihrem Ausflicken hinweisen. Wir haben berechtigten Grund zur Annahme eines eigentlichen Tiefseefanges mit der langen Leinc, da im jungsteinzeitlichen Saisonplatz Ruskenesset der Leng (Molva) in großer Zahl gefischt wurde, der sich meist in Tiefen von etwa 100 bis $300 \mathrm{~m}$ aufhält.

Schon in dieser frühen Zeit erscheint die norwegische Küste als ein hervorragender Meerfischplatz in Europa. Gleichzeitig muß sich der Fang bis ins Nordland ausgedehnt haben. Wie wir sahen, war der Kabeljaufang stets eine mit dem Bauerntum kombinierte Wirtschaftsform.

Die Bevölkerungszunahme und die Verstädterung in Mittel- und Südeuropa schufen die Voraussetzungen für einen aufnahmefähigen Markt. Damit ging Norwegen im Mittelalter von dem für lokale Bedürfnisse betriebenen Fischfang zum eigentlichen Groß-Fischfang und zu einem großzügig betriebenen Fischhandel über.

Die alten Methoden der Fischkonservierung sind das Dörren und Salzen. Die zum Dörren bestimmten Fische halbiert man bis zur Flosse und hängt sie auf über $2 \mathrm{~m}$ hohe Holzgestelle zum Trocknen auf. Hier hängen bis in den Sommer hinein die Stock-Fische zu Hunderttausenden. Dann werden sie heruntergeholt, zu Ballen zusammengebunden und versandt. Anders werden die Klippfische zubereitet, die man zuerst salzt und dann auf kahlen Felsen zum Trocknen auslegt. Die heutige Ausfuhr von Stockfisch und Klippfisch hat ihren Ursprung im Mittelalter. Damals a $\beta$ man in der ganzen christlichen Welt am Freitag Fische. Da frische Fische in den südlichen Breiten früher nicht erhältlich waren, wurden die norwegischen Trockenfische in großen Mengen importiert, vor allem von lateinischen Ländern, wie Portugal, Spanien, später auch in Brasilien, Argentinien und Kuba.

Wenn der Kabeljaufang auf eine uralte Vergangenheit zurückblicken kann, so ist die Heringfischerei in der ganzen Urgeschichte Norwegens nur einmal und nur mit einem Knochen in der Solsemhöhle bei Leka nachweisbar. Während dieser Fund wohl bronzezeitlich ist, finden sich Reste des Herings in der dänischen Steinzeit. Der Hering spielte aber erst im Mittelalter eine wirkliche Rolle, als es galt, die in den Städten zunehmende Bevölkerung mit einem billigen Nahrungsmittel zu versorgen.

Heute ist die Fischereiindustrie vielseitiger. Der Mittelpunkt einer Konservenindustrie liegt bei Stavanger. Neuzeitliche Kühlmethoden mit Eis und dem Tiefgefrierverfahren erlauben den Versand von frischen Fischen, aber auch Delikatessen, wie Hummer und Krebsen. Aus der Dorschleber wird immer noch der Lebertran gewonnen. Die Fischblase liefert den Fischleim und aus Rogen stellt man Kaviar her. Nebenprodukte der Fischerei sind auch Heringöl und Fischmehl. Die Fischköpfe lassen sich an die Haustiere des norwegischen «Bauernfischers 》 verfüttern.

\title{
BEWÄSSERUNGSANLAGEN AM NIGER IN FRANZÖSISCH WESTAFRIKA
}

\author{
ERNST LeEMANN
}

\section{Ü BERS I CHT}

Geographische Orientierung. Wenn man kurz vor der Landung auf dem interkontinentalen Flugplatz Bamako im französischen Sudan den grün-blau schimmernden Niger überfliegt, erkennt man trotz der in der Trockenzeit stark reduzierten Wassermenge den imposanten Charakter dieses afrikanischen Flusses. 


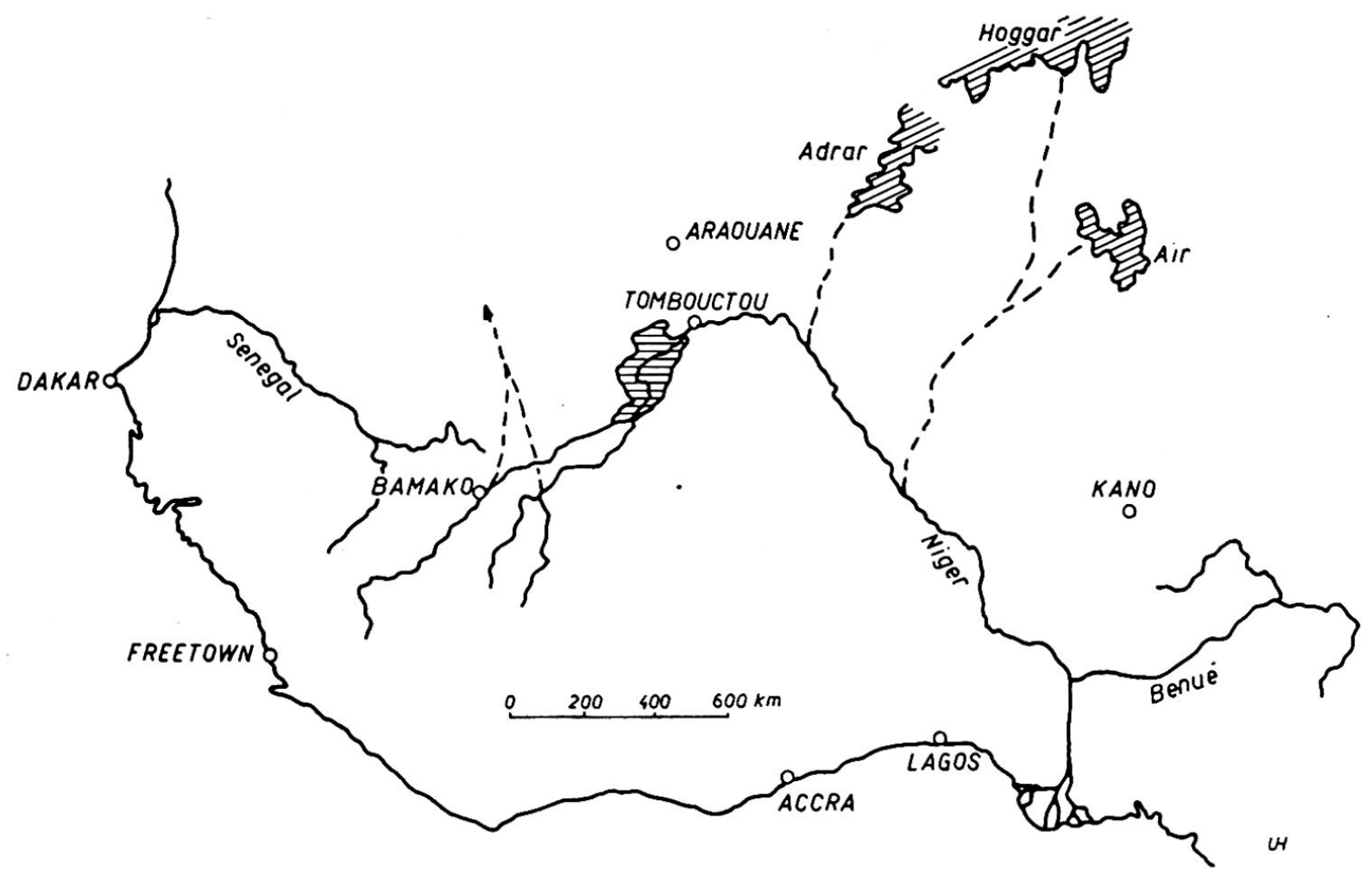

Fig. 1. Entwicklung des Flußnetzes; Ablenkung des Oberlaufes des Niger durch hauptsächlich östliche Flüsse

Zahllose, grasbewachsene Inselchen ragen aus dem träge fließenden Wasser hervor und großflächige Sandbänke füllen nicht selten das halbe Flußbett aus oder tauchen als dunkelgelbe Flecken nahe zur Wasseroberfläche empor. Es ist offenbar, daß die Schiffahrt, die allerdings nur während der Hochwasserzeit in größerm Maße möglich ist, nur mit ortskundigen Steuerleuten betrieben werden kann. Selbst die langgestreckten, schmalen und flachbodigen Pirogen der Eingebornen suchen sich mühsam den Weg zwischen den sandigen Sperren hindurch.

Die Hochwasserstände sind bedeutend; sie liegen bei Sansanding normalerweise fünf Meter über dem Niederwasser. In der Trockenzeit beträgt die Abflußmenge hier rund $50 \mathrm{qbm} / \mathrm{sec}$; in der Regenzeit steigt sie bis $6000 \mathrm{qbm} / \mathrm{sec}$. an.

Bis Sansanding fließt der Niger in einem ziemlich geschlossenen, aber wenig eingetieften Tal, dessen Breite er in großen Mäandern voll ausnützt. Bei Bamako säu men die Berge von Mandingo die linke Talflanke als scharf ausgebildetes Prallufer, bald aber treten nur noch wenige Restberge von bescheidener Höhe aus den weiten Ebenen, die sich in weichen Formen in der Ferne verlieren. Schwarze, rauchende Flächen lassen selbst aus großer Höhe die immer wieder angewandte Brandmethode des Eingebornen in der Brousse, die der Niger hier durchzieht, erkennen und kleine, gelbliche Rechteckflächen verraten den dürftigen Ackerbau, der außerhalb der künstlichen Bewässerungszonen mit recht wechselndem Erfolg betrieben wird. Flußabwärts gegen Ségou verschwinden alle Unebenheiten; unübersehbare Ebenen, dürftig bewachsen, geben dem Fluß ihr eintöniges Geleit. Bis Tombouktou trägt der Niger bei den Eingebornen den Namen Dioliba, den mittlern Teil bis Gao nennen sie Issa-Ber und den eigentlichen Unterlauf Kouarra.

Historische Verhältnisse. Die Geschichte des Nigers ist nicht eindeutig bekannt, doch gelten einige bedeutsame Flußverlegungen als gesicherte Tatsachen. Von französischen Geographen vermutet, bis heute aber nicht einwandfrei nachgewiesen, ist eine Vereinigung des Nigers mit dem Senegal, dessen obere Zuflüsse den Weg westlich Sokolo gegen das Hodh genommen haben sollen. In einem spätern Zustand mündete der Niger im Sudan in einen großen, saharischen See. Nach HuBERT ergoß er sich in einem westlichen Arm in die Region von Niafunké und Goundam, in einem östlichen von Sansanding gegen Diafarabé. Vom Nordrand dieser tschadseeähnlichen Seen- 


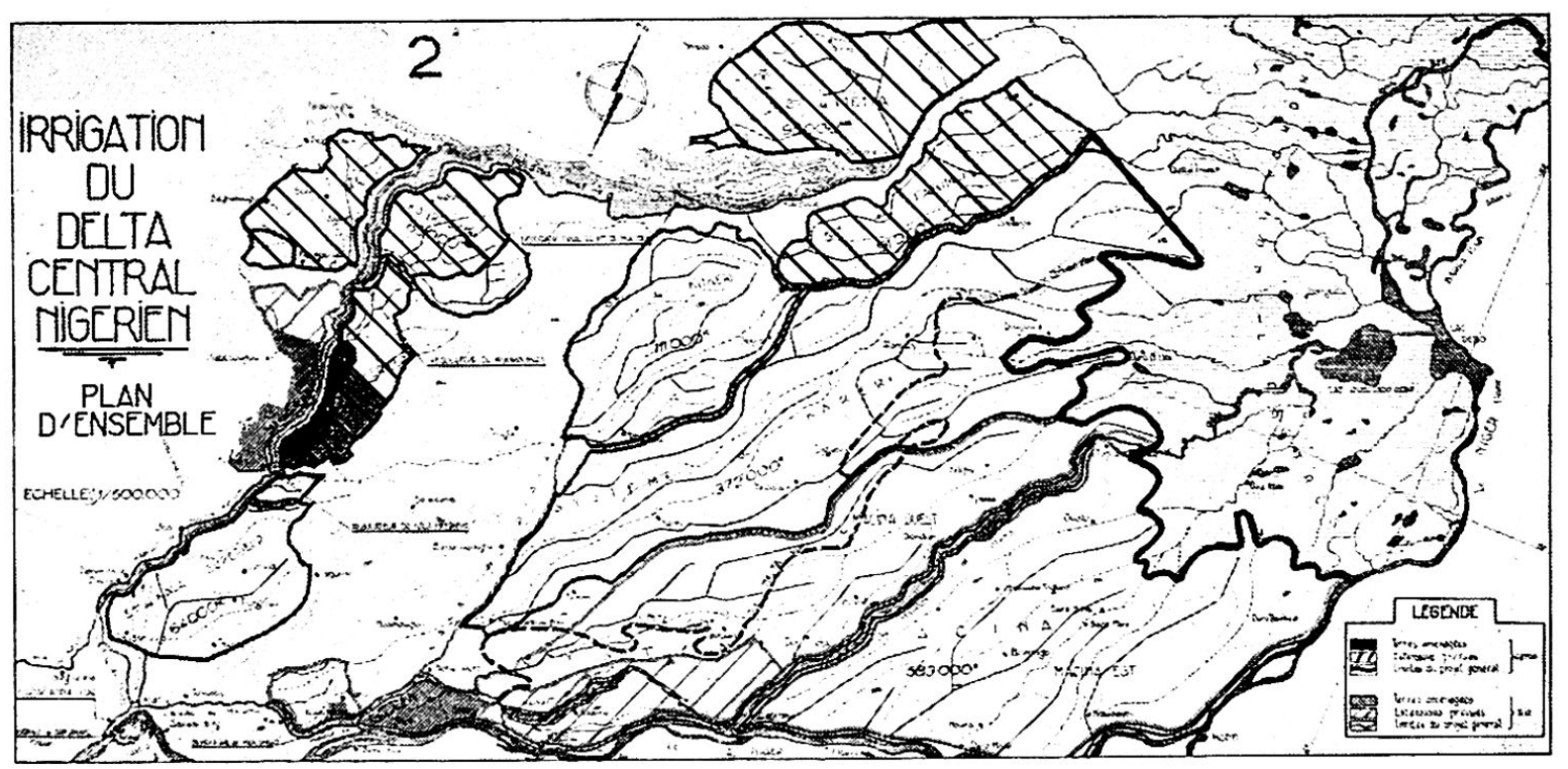

Fig. 2. Ehemaliges Nigerdelta am Südrand der Sahara

Gestrichelte Flächen: Baumwollbauzonen; Weiße Flächen: Reisbauzonen; Schwarze und graue Flächen: Bereits in Betrieb genommene Teile. Dicke Randlinie: Grenzen des vorläufigen Projektes

zone, die mit dem heutigen Hochwasser-Überschwemmungsgebiet ordentlich übereinstimmen soll, floß Nigerwasser bei Fagibine und Tombouctou weiter nordwärts in die Sahara hinein. Diese Auffassung wird gestützt durch alte Spuren eines hydrographischen Systems bei den Oasen von Araouan, wo die Brunnen auch heute regelmäßig auf das Nigerhochwasser reagieren; außerdem kennt man Überlieferungen eingeborner Stämme, die historisch untermauert sind. Es steht heute fest, da $\beta \mathrm{im}$ 1. Jahrhundert unserer Zeitrechnung nördlich des jetzigen Nigertales das bedeutende Reich von Ghana bestand, das im Besitze hoher Kultur war. Im 11. Jh. ging dieses Reich endgültig unter; als Gründe werden genannt: fortschreitende, unaufhaltsame Austrocknung des Bodens und Einbruch des Islams.

Der Niger floß also wohl bis gegen das 10./11. Jh. unterhalb Bamako nord- und nordostwärts der Sahara zu, bis ihm mächtige Sanddünenzonen, die ja vom Atlantik bis zum Tschadsee ziehen, den Weg versperrten und ihn zum Versanden brachten. Erst als der ehemalige Azaouak, der seine Wasser in den Bergländern des Adrar des Iforas und des Air sammelte und ab Bikini den Unterlauf des heutigen Nigers darstellt, rückwärts einschnitt, kam es zur Anzapfung des bisherigen Nigerlaufes und damit zur Ausbildung des heutigen Flußsystemes. Mit der Ablenkung aber wurde einer gut bevölkerten Zone das lebenswichtige Wasser langsam, aber endgültig entzogen. Große Ländereien vertrockneten, wurden zu Wüste und ihre bisherigen Bewohner, reich an Zahl, verloren jegliche Existenzmöglichkeit in ihren angestammten Wohngebieten. Wohl haben wir auch heute noch in südlichern und tiefern Talauen wenigstens während der Hochwasserperioden Überschwemmungsflächen, die etwelches Leben ermöglichen, aber die verschiedenen, zahlreichen Seen von heute sind nur geringe Reste der einstmals bewässerten Gegend. So bestehen heute zwei Deltazonen : eine lebende, die auch heute während der Hochwasser überschwemmt wird, und eine tote, deren alte Flußläufe hoch liegen und darum trocken bleiben; beide zusammen bilden das "delta central nigérien", das durch die großartigen Arbeiten des Office du Niger erneut bewässert und dem Anbau und damit der Besiedlung zugeführt wird. Bevor man sich jedoch an das große Projekt heranwagte, suchte man auf zwei kleineren Versuchsfeldern Erfahrungen zu sammeln.

Erste Versuche. 1922 wurden am rechten Ufer des Nigers bei Niénébalé gegenüber von Koulikoro die ersten Versuche angestellt. Mit 1500 zugezogenen Eingebornen, denen man das bestellte Land bald als Familienbesitz überließ, wurden 1500 ha Land nach gegebenen Anordnungen bestellt und bald hohe Erträge erzielt. Schon sieben Jahre später wurden diese Versuche bei Bagninéda auf breiterer Basis weiter geführt, nachdem man bei Sotuba, zwei Kilometer unterhalb Bamako, einen Staudamm und flußabwärts einen $22 \mathrm{~km}$ langen Bewässerungskanal errichtet hatte. Durch diese Maßnahmen wurden 3000 ha für Reisbau, 3000 ha für Baumwoll-, Mais-, Maniok- und Erdnußbau und 1500 ha für Viehweide gewonnen. In 15 Dörfern wurden 5600 Eingeborne vom Stamme der Bambara angesiedelt. Die Ergebnisse dieses zweiten Versuches befriedigten in hohem Maße, so $\mathrm{da}$ man jetzt, mit umfassenden Erfahrungen in technischer, landwirtschaftlicher und soziologischer Hinsicht ausgerüstet, an das große Werk im zentralen Nigerdelta herangehen konnte.

Grïndung des Office du Niger. Am 5. Januar 1932 wurde das Office du Niger durch Regierungsdekret offiziell konstituiert „pour objet d'étude, l'aménagement et la mise en valeur de la vallée du 


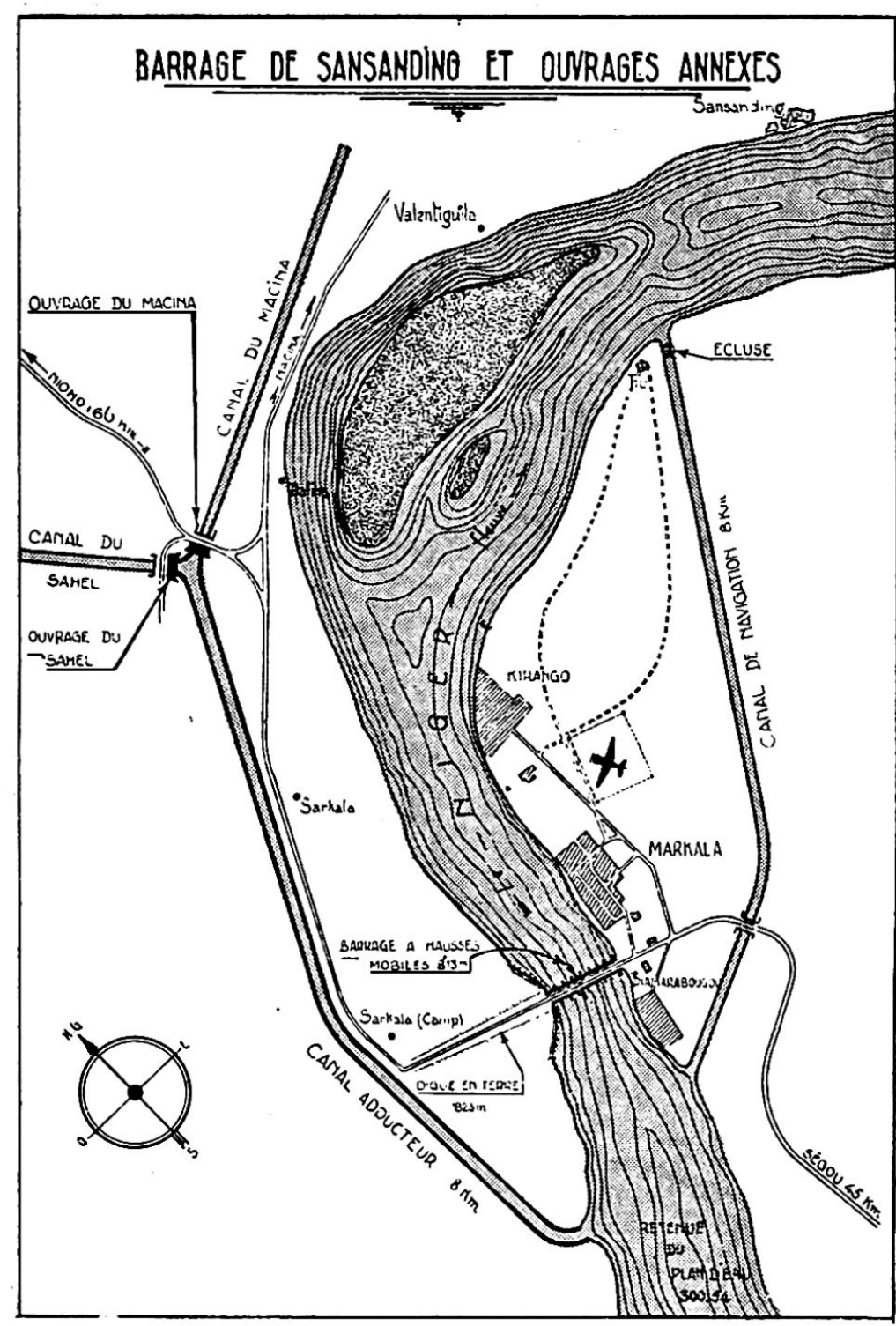

Fig. 3. Lage des Staudammes und der Hauptkanäle bei Sansanding
Niger". Damit wurde das Office du Niger zu einer öffentlichen Unternehmung mit autonomer Finanzverwaltung. Oberste Instanz ist ein Administrativrat, die Leitung besorgt ein Generaldirektor mit Sitz in Ségou, wo auch die gesamte Verwaltung in einer besondern Konzession angesiedelt ist.

Planung, Verwaltung, Ausbau der Bewässerungsanlagen, Studien über Anbaumöglichkeiten, Kolonisierung der neu gewonnenen Territorien, Anleitung und Erziehung der Kolonisten, Übernahme und Verkauf der Ernten, alles, was mit einer solch großen Unternehmung zusammenhängt, ist Aufgabe des Office.

Grund und Boden seiner Unternehmungen sind Eigentum des französischen Staates, der ja auch das ganze Unternehmen finanziert und alle Risiken trägt. 1)ie Eingebornen, die sich auf diesen ehemals wüstenhaften Territorien niederlassen, um das Land zu bebauen, erhalten nach zehn Jahren der Bewährung das Recht der dauernden Nutznießung und dessen Vererbung auf ihre Nachkommen. Der Eingeborne befindet sich also in einem Pachtverhältnis zum Staat, der ihm Land, Wasser, Wohnung, Vieh und Werkzeug zur Verfügung stellt gegen einen bescheidenen Pachtzins, der in Naturalien beglichen wird.

\section{TECHNISCHE GRUNDLAGEN}

Die bereits im Jahre 1924 begonnenen kartographischen Arbeiten bestimmten die Führung der Bewässerungskanäle. Erleichtert wurde deren Anlage durch das Absinken des Landes auf der linken Nigerseite, das bis zum $120 \mathrm{~km}$ entfernten Sokolo rund 10 Meter tiefer liegt. Bei diesen Projektierungsarbeiten entdeckte man zwei alte Flußarme, deren Enden mit Flußgeschieben und Triebsand verstopft waren. Der eine dieser Arme lag parallel zum jetzigen Flußlauf und verlor sich nach ungefähr $200 \mathrm{~km}$ im Gebiet von Macina, der andere drang direkt nordwärts gegen das Sahel vor.

Um das Berieselungswasser auf die nötige Höhe zu bringen, wurde der Staudamm von Sansanding erbaut. Vom rechten Flußufer aus quert ein moderner Regulierdamm von $816 \mathrm{~m}$ Länge den Flu $\beta$ und wird von einem festen Erddamm ohne Durchlässe um weitere $1800 \mathrm{~m}$ fortgesetzt, soda $\beta$ das ganze Hochwasserbett auf einer Breite von $2600 \mathrm{~m}$ gesperrt wird. Durch 14 Durchlässe wird der Flut Abfluß ermöglicht. Die Stauung reicht trotz der mächtigen Breite des Flusses weit über das $32 \mathrm{~km}$ oberhalb des Dammes liegende Ségou hinauf. Die Stauhöhe von $5 \mathrm{~m}$ über das Niederwasser garantiert während sieben Monaten genügende Bewässerung der in Aussicht genommenen Flächen. 
Fig. 4. Staudamm mit Wasserdurchlässen und Straßenbrücke. Konstruktive. Schwierigkeiten bereitete der Durchlaß der Hochwasserflut. Die Brücke kann auch als Bahnübergang benützt werden

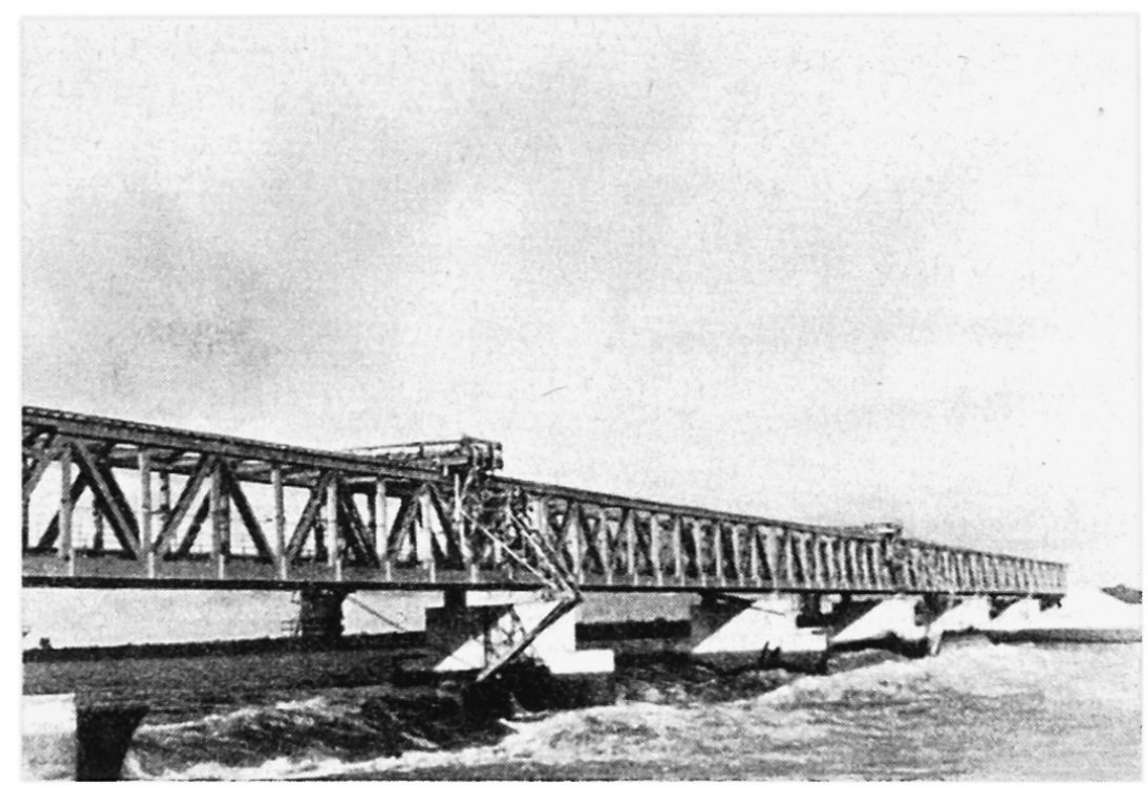

Das natürliche Niederwasser des Flusses in den Monaten Februar bis Juni fällt in die Zeit des Aussetzens der Bewässerungsnotwendigkeit, was die geringe Stauhöhe erklären mag. Der Damm dient gleichzeitig als Straßenbrücke, könnte aber im Bedarfsfalle auch ein Bahntrasse tragen. Ein Schiffahrtskanal von $8 \mathrm{~km}$ Länge überwindet mit Hilfe einer 53 m langen Schleuse die Staustufe. Eine Dieselzentrale, deren Motoren von Sulzer in Winterthur stammen, erzeugt $2000 \mathrm{Kw}$ elektrischen Strom, der zur Bedienung der Schützen, zu gewerblichen Zwecken in den Werkstätten und zu Beleuchtungszwecken in der Konzession dient.

Der im Jahre 1934 begonnene Bau des Dammes war 1948 vollendet. Seine Fertigstellung wurde durch die Auswirkungen des Krieges stark verzögert. Ein Zuleitungskanal gibt das Wasser nach $8 \mathrm{~km}$ Lauf an die beiden Hauptbewässerungsadern des ganzen Systems ab, an den Canal du Sahel und den Canal de Macina. Die Zuteilung wird durch eingebaute Regulierwerke geregelt; im Maximum fließen 500 qbm/sec. durch, was ungefähr der halben Abflußmenge des Rheines bei Basel entspricht! Beide Kanäle erreichen nach rund $20 \mathrm{~km}$ Lauf die vorhin erwähnten alten Flußläufe, den «Fala de Molodo» und «la rivière de Boky-Wéré », denen sie in die Bewässerungsgebiete folgen können.

Um die dem Hauptfluß zunächst gelegenen Berieselungsfelder vor den wilden Hochwassern zu schützen, wurde linksufrig ein $69 \mathrm{~km}$ langer Deich gebaut, der den Hochwassern bis heute standgehalten hat. Besonders bei Hochflut dient er auch als begehrter Weg. Die heute erstellten Zufuhr- und Bewässerungskanäle erreichen eine Gesamtlänge von $3182 \mathrm{~km}$ und verursachten eine Erdbewegung von 25 Millionen Kubikmetern! Erst beim realen Anblick der verwirklichten Anlagen und Bauten an Ort und Stelle, inmitten einer $1500 \mathrm{~km}$ von der Küste entfernten Halbwüste, vermag man die vollbrachte Leistung voll und ganz zu würdigen. Neben der Schaffung der eigentlichen Bewässerungsanlagen waren Wohnhäuser, Magazine, Depots, Garagen, Werkstätten, Wasser- und Elektrizitätswerke, Ziegeleien, Reismühlen, Entkernungsanlagen, Öl- und Seifenfabriken, Verkehrswege und Fahrzeuge zu erstellen und unzählige weitere Aufgaben zu bewältigen, sodaß es einer unendlichen Ausdauer und eines nie erlahmenden Glaubens an das gute Gelingen der großen Aufgabe bedurfte. Vollständige Hingabe an das beabsichtigte Werk und eine große Begabung führten es zur heutigen Blüte.

All das aber war nur möglich, weil der Glaube ans Werk auch die finanziellen Quellen zu erschließen vermochte. Der französische Staat investierte am Niger riesige Kapitalien während langen Jahren, denn erst in den allerletzten Jahren vermochte 
sich die Unternehmung selbst zu tragen. Die vergangenen Jahrzehnte haben bewiesen, daß die Worte des jetzigen Generaldirektors des Office du Niger, Georges Peter, voll und ganz der Wahrheit entsprechen, wenn er sagte, daß Frankreich am Niger nicht in erster Linie ein rentables Unternehmen gründen wollte, sondern daß es die Lebensmöglichkeiten der Schwarzen verbessern wollte durch die Ertragssteigerung lebenswichtiger Produkte.

Anfänglich war projektiert, 510000 ha Land mit Baumwolle, 450000 ha mit Reis zu bepflanzen, total also eine Fläche, die gut $2 / 3$ des schweizerischen Mittellandes umfaßt. Bis Ende 1953 waren allerdings erst 38500 ha unter dem Pflug, denn das Fortschreiten der Arbeiten ist durch den Krieg sehr stark behindert worden.

Die Ernte 1952/53 zeitigte erfreuliche Ergebnisse; 34890 t Reis und 2450 t Baumwolle konnten eingebracht werden. Die Hektarerträge für Reis sind allgemein hoch, wenn auch schwankend; sie betrugen im Sektor von Baguinéda $1300 \mathrm{~kg}$, in Molodo jedoch $2100 \mathrm{~kg}$, während für das Mekongdelta $1100 \mathrm{~kg}$ angegeben werden.

\section{SIEDLUNG UND WIRTSCHAFT}

Ansiedlung der Kolonisten. Ein besonderes Problem bildet in großen Teilen Afrikas die Gewinnung einer genügenden Anzahl brauchbarer Arbeitskräfte, da die Umsiedlung größerer Menschenmengen hier zahlreichen und recht verschiedenartigen Hindernissen begegnet. Ohne ausreichende Arbeitskräfte sind alle technischen Vorkehren sinnlos, denn die endgültige Nutzbarmachung neu erschlossener Ländereien ist der Tätigkeit des Menschen vorbehalten.

In den Versuchsgebieten von Niénébalé und Baguinéda, in denen Eingeborne als Kolonisten angesiedelt wurden, stellte man bald fest, daß ersprießliche Resultate durch Einführung europäischer Angestellten- und Lohnverhältnisse unmöglich waren. Erst als man die Schwarzen durch den Familienbesitz des zu bebauenden Bodens am Ertrag interessierte, begannen sich Erfolge einzustellen. Trotzdem bedarf es auch bei diesem Prinzip sorgfältiger Führung in technischer und agronomischer Hinsicht. Für die erfolgreiche Erschließung aber kommt der Eingeborne nur in Frage, wenn er mit seiner ganzen Familie an Grund und Boden und an den Erträgen interessiert werden kann und ihm dann die neuen Wohngebiete auch zur neuen Heimat werden. Das aber hat neben der Regelung der materiellen Grundlagen auch sorgfältige Planung der sozialen Verhältnisse und die Berücksichtigung der menschlichen Eigenarten in all ihren mannigfaltigen Nuancen zur Voraussetzung. Wie groß gerade solche Schwierigkeiten in diesen Gebieten sein können, zeigt J. GaBUs in seinem Aufsatz: «La Colonisation chez les Touareg de la boucle du Niger» (Acta Tropica, Basel, 1945).

Die neuen Kolonisten müssen in ziemlich entlegenen Gebieten gewonnen werden, da das Nigerland ja sehr schwach besiedelt ist. Während in den Anfängen der Neubesiedlung eher zu wenig Kolonisten zu finden waren, ist heute die Placierungsmöglichkeit kleiner als das jeweilige Angebot, umsomehr, als man jetzt weiß, daß das bewässerte Land maximal 100 Menschen pro $\mathrm{qkm}$ zu tragen vermag.

Die Besiedlung der neu gewonnenen Gebiete bietet aber auch in persönlicher Richtung allerlei Schwierigkeiten, denn der Kolonist muß sich in mancher Beziehung innerlich und äußerlich umstellen. Oft müssen liebgewordene Sitten und Bräuche abgestreift werden, weil sie mit den neuen Pflichten nicht mehr vereinbar sind oder gar in Widerspruch geraten. Zudem hat der Schwarze ohne Zweifel in seiner neuen Stellung viel Neues zu erlernen und zu begreifen, selbst wenn ihm bisher sogar der Reisbau oder andere Bodenbearbeitungen nicht fremd waren. Er muß die Handhabung moderner Pflüge und Eggen lernen und sich auf den Umgang mit Zugtieren einstellen. Nicht selten hat er zum ersten $\mathrm{Mal}$ in seinem Leben Vieh zu füttern und zu pflegen, Felder für die Aussaat bereit zu stellen, Saatpflege zu treiben und schließlich die Ernte einzubringen. $Z$ wischen all dem liegen tausend andere, auch nicht unwichtige Dinge, in denen der Neuling, der meist im guten Mannesalter steht, Hilfe und 
Fig. 5. „La Brousse“. Zustand der Deltazone vor Arbeitsbeginn. Hartes Steppengras, dornige Akazien und ärmliche Gummibäume bedecken den Boden

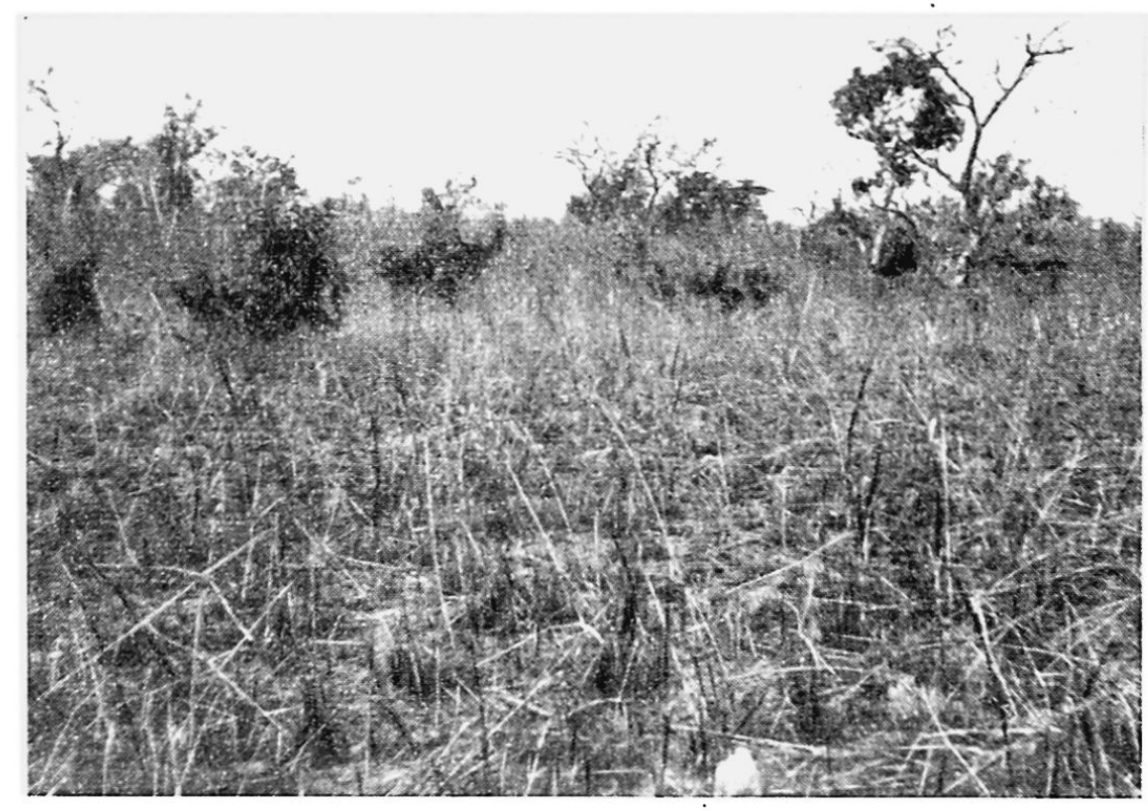

Anleitung oder mindestens Rat benötigt. All das aber muß mit Feingefühl und Einfühlungsgabe besorgt werden, wenn der Eingeborne Zutrauen zum Weißen und seiner Sache und damit auch Geborgenheit in der neuen Situation finden soll. Und erst wenn es endlich so weit gekommen ist, wird der Eingeborene Sicherheit und Selbständigkeit in seiner neuen Arbeit finden können. Darum sind auch die Übereinkommen zwischen dem Office du Niger und den Eingebornen von großem Verständnis für die Eigenart und die Fähigkeiten des schwarzen Kolonisten getragen; sie haben denn auch in den letzten Jahren ausgezeichnete Erfolge gezeitigt.

Die Zuteilung des Bodens erfolgt stets an den Familienchef, da ja nur er handlungsberechtigt ist. Sie beträgt normalerweise $4-6$ ha, jedenfalls nie mehr, als mit familieneigenen Kräften bewirtschaftet werden kann. Wohnung, Arbeitsausrüstung, Vieh (1 Milchkuh, 4 Zugochsen), Vorräte und Nahrungsmittel für den Anfang werden zur Verfügung gestellt. Auch für die Versorgung auf der Herreise wird gesorgt.

Die Kolonisten werden in Dörfern angesiedelt, wo sie aber ihr dörfliches Eigenleben vollständig selbst bestimmen. Eine ziemlich große Schwierigkeit liegt in der Versorgung mit gutem Wasser, doch konnte man mit den heutigen technischen Möglichkeiten auch hier das Ziel erreichen. Nur langsam nach und nach, beginnt der Eingeborne sein sauberes und gesund angelegtes Dorf zu lieben und lernt auch seine bisherige Eigenart, die in der Brousse draußen durch nichts eingeschränkt war, den neuen Verhältnissen einigermaßen anzupassen und entsprechend $\mathrm{zu}$ formen. Auch hier genießt er vom Office aus alle denkbare und sinnvolle Unterstützung.

Ökonomische Situation. Die Anteile an den Ernteerträgen sind reichlich, sodaß alle Familien gut leben können. Sie stellten sich in jeder Beziehung viel besser als in ihren frühern Verhältnissen. Nachstehende Zahlen belegen diese Tatsachen:

\section{Mittlerer Reisertrag pro Jahr}

Abzüge: Für Steuern

Für Genossenschaft

Für Office (Maschinen)

Für Dreschen

Total der Abzüge

Verfügbare Menge

Davon gehen ab für Saatgut

Davon gehen ab für Ernährung

Für Verkauf bleiben übrig

Barerlös

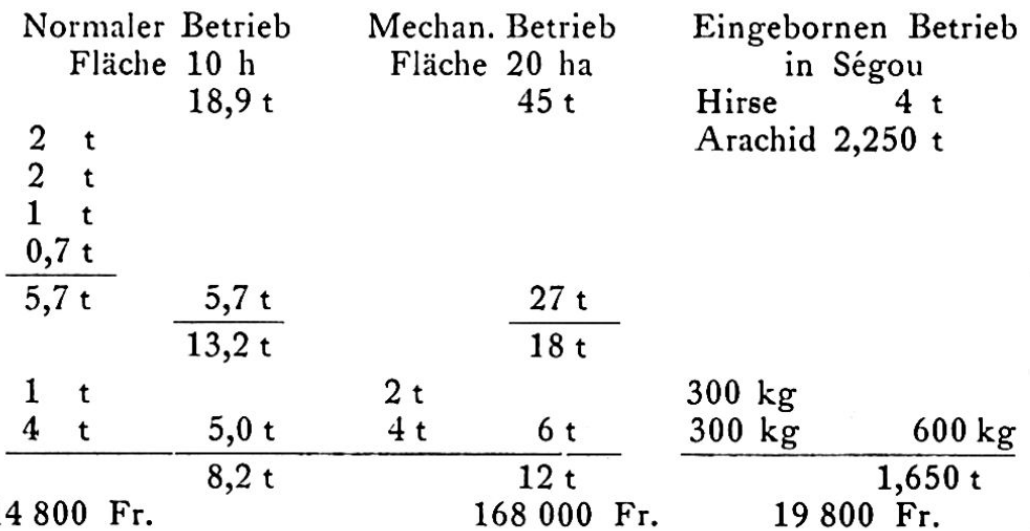




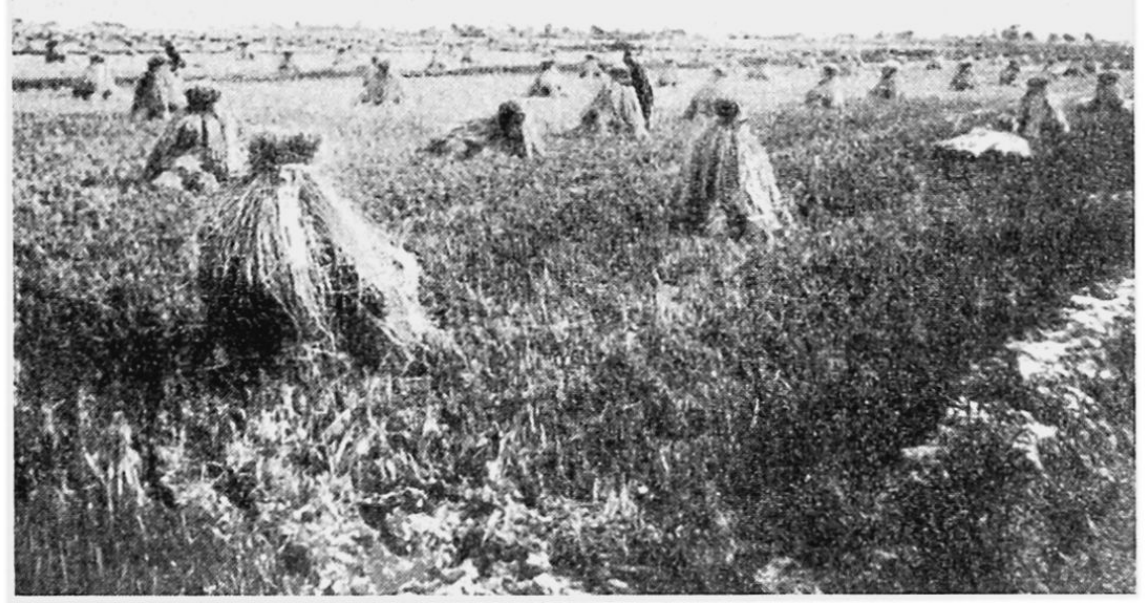

Fig. 6. Abgeerntete Reisfelder bei Boky Wéré. Die Ernte wird mit Maschinen eingebracht. Für ihre Verarbeitung werden Saisonarbeiter aus Frankreich zugezogen.

Diese Zahlen belegen ganz einwandfrei den Vorteil, den die Kolonisten auf den Territorien des Office haben; sie lassen aber auch erkennen, daß der mechanisierte Betrieb die größten Erfolge ermöglicht. Auch zeigen diese Zahlen, daß das Office recht unbedeutende Abzüge als Gegenleistung verlangt.

Um den Nutzeffekt weiterhin zu erhöhen, wurden landwirtschaftliche Genossenschaften gegründet, deren Mitglieder sich aus den Chefs der Familien rekrutieren. Hauptaufgabe dieser Vereinigungen ist die Verbesserung der Familiendomäne und ihrer Ausbeutung. In diesem Sinne sind der Genossenschaft viele Arbeiten überbunden, wie Unterhalt und Verbesserung der Bewässerungsanlagen, Hausbau, Pflanzen von Bäumen im Dorf, Unterhalt und Verbesserung der Weganlagen innerhalb ihres eigenen Zentrums. Sodann besorgt die Genossenschaft den Verkauf von Produkten für den persönlichen Bedarf: Stoffe, Haushaltungsgegenstände, Lebensmittel und anderes. Aber auch der Verkauf der verfügbaren Ernteerträge erfolgt durch die Genossenschaft. Diese kann ihre Mitglieder auch finanziell unterstützen durch Kredite auf Termin oder durch Erntevorschüsse. Um Fehlentwicklungen zu vermeiden, hat die Regierung in diesen Organisationen eine Reihe zweckmäßiger Sicherungen eingebaut.

Herkunft der Bevölkerung. Trotzdem es im Gebiet des Macina einige größere Siedlungen gab, deren Lebensmöglichkeit auf den jährlichen Überschwemmungen des Flusses beruhten, reichten diese ansässigen Schwarzen bei weitem nicht aus zur Kolonisierung der neu erschlossenen Ländereien. Da aber die umgebende Sahelzone nur dünn bevölkert ist, war man genötigt, aus recht abliegenden Gebieten Leute herbei zu ziehen. Es waren hauptsächlich Bambara aus dem Gebiet von Ké-Macina, Ségou und Bamako, Minianka von San und Koutiaba, Marka von Tougan und Mossi aus dem fernern Süden. Die Heimat der Minianka liegt $150 \mathrm{~km}$ entfernt, diejenige der Marka $350 \mathrm{~km}$, und die Mossi kamen über $450 \mathrm{~km}$ weit her. Da sie aber alle ähnlichen Klimagebieten entstammten, akklimatisierten sie sich im neuen Wohngebiet sehr schnell. Während in den ersten Jahren der Neubesiedlung des Nigergebietes auch Unfreiwillige durch entsprechende Administrativmaßnahmen zwangsweise hieher verpflanzt wurden, die sich begreiflicherweise wenig bewährten, war die Kolonisation im Delta nach wenigen Jahren so populär geworden, daß man von solchen Maßnahmen restlos Umgang nehmen konnte. Trotzdem gibt es auch heute dann und wann Unzufriedene oder Enttäuschte, die darum wieder wegziehen. Andere wieder gehen in ihre alte Heimat zurück, um dort die im Delta gemachten Ersparnisse zu verbrauchen. Später kehren sie reuig wieder zurück. 
Fig. 7. Reisspeicher der Eingeborenen in einem Kolonistendorf. Jede Rassengruppe bewohnt ihr eigenes Dorf. Große Sorgfalt ist den hygienischen Einrichtungen zuteil geworden. Die umfänglichen Reisspeicher enthalten Saatgut und Nahrungsreserven.

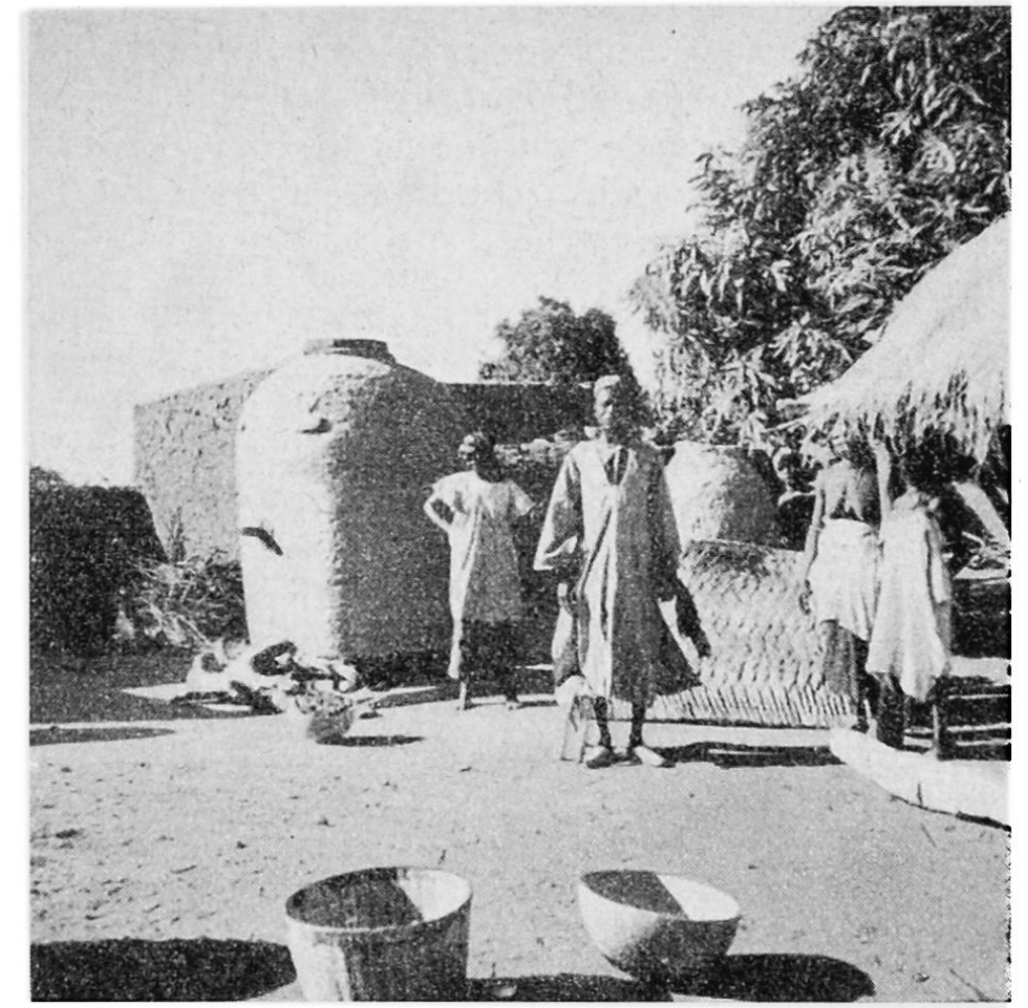

Betreuung der Kolonisten. Stammesunterschiede, religiöse Differenzen, Marktleben und Feste bringen weiterhin ab und zu schwierige Situationen, denen die Organe des Office begegnen müssen. Selbst die Freizeit stellt Probleme verschiedener Art.

Wie bereits bemerkt, bemüht sich das Office um das Wohlergehen der Eingebornen in vorbildlicher Weise. So liegen ihm auch die sanitären Aufgaben sehr am Herzen. Das zeigt vor allem ein Zahlenvergleich in eklatanter Weise. Das französische Gouvernement gibt im Sudan pro Kopf und Jahr für solche Zwecke $110 \mathrm{Fr}$. aus; im Bereiche des Office werden für die gleichen Aufgaben jedoch im selben Zeitraum 1466 Fr. aufgewendet! Scharf wird der Kampf besonders gegen die Malaria, die für die große Kindersterblichkeit verantwortlich sein soll, geführt. Neben der Bekämpfung der Krankheitsübertragung wird auch den prophylaktischen Maßnahmen bei Kindern und Erwachsenen alle Aufmerksamkeit geschenkt. Man hat erreicht, daß der Gesundheitszustand der Kolonisten recht gut ist. Regelmäßige ärztliche Überwachung, Einrichtung medizinischer Posten in den einzelnen Zentren und ein modern ausgestattetes Spital in Markala sind Garanten gesunder Lebensmöglichkeiten.

Die Erziehung der Eingebornen zu vernünftiger Lebensweise gehört $\mathrm{zu}$ den schwierigsten Aufgaben des Office, denn der Schwarze ist immer noch der Gefangene althergebrachter Anschauungen und Gebräuche und sein Sinn ist noch voll von fetischistischem Glauben.

Seit 1946 hat jeder Kolonist völlige Bewegungsfreiheit. Man war bei deren Einführung auf eine gewisse Abwanderung gefaßt, doch nichts dergleichen trat ein! Die Bevölkerungsbewegung ist in jeder Beziehung normal, und steigende Ziffern beweisen die Beliebtheit der neuen Territorien. Im Jahr 1942 waren 25564 Menschen angesiedelt, 1946 waren es 29425.

Landwirtschaftliche Produktion. Die Baumwollproduktion hat innerhalb der französischen Union sehr entwicklungsfähige Aussichten, denn der allergrößte Teil der Rohbaumwolle für die hochentwickelte Textilindustrie muß aus fremden Ländern eingeführt werden. Die Steigerung der Eigenproduktion ist für den Finanzhaushalt 
des Mutterlandes auch devisenpolitisch bedeutend. Sodann sind die in Dakar neugegründeten Textilfabriken natürlich gerne Abnehmer der Nigerbaumwolle. Detaillierte Bodenuntersuchungen haben ergeben, daß im Gebiet von Kouroumari für die langfaserige Baumwolle recht günstige Bedingungen bestehen, während sich die Böden der Kala-Inferieur eher für amerikanische Sorten eignen.

Der Reisbau ist meist völlig getrennt vom Anbau der Baumwolle, da er ganz andere Bedingungen zur Voraussetzung hat. Zahlreiche Sorten sind auf ihre Eignung im Soudan geprüft worden. Vorab amerikanische Sorten sind hier wegen geringen Erträgen ungeeignet. Der Reis wird weniger im Mutterland, als im französischen Westafrika benötigt, besonders in Senegalesien, wo durch etwas einseitige Produktionslenkung für Exportwaren nun die einheimische Versorgung nicht mehr zu genügen vermag. Zudem ist immer deutlicher zu beobachten, daß sich die Eingebornen von der bisher im Vordergrund stehenden Hirse abwenden und mehr und mehr den Reis bevorzugen.

Alljährlich werden rund $60000 \mathrm{t}$ Reis nach Westafrika importiert und müssen mit teuren Devisen bezahlt werden. Für den Reisbau am Niger eröffnen sich somit die besten Perspektiven.

\section{AUSSICHTEN}

Das Werk am Niger hat große Teile der unfruchtbaren Brousse in edles Kulturland verwandelt. Einer riesigen Oase gleich dehnen sich heute seine Flächen aus. Wo vor weniger Dezennien noch kaum kümmerliches Leben möglich war, wo verderbliche Seuchen grassierten und nicht selten Hungersnot zu Gaste war, blüht neues, intensives und glückliches Leben. Nicht Profit wurde in erster Linie angestrebt, sondern humane Kolonisation. Das ganze Werk ist in allen Teilen wohl bedacht und hat ohne Zweifel eine sichere Zukunft vor sich, denn es ist auf Menschlichkeit gegründet. Noch steht es in den Anfängen im Hinblick auf seine immensen Möglichkeiten. Es ist nicht ein Werk von Heute, sondern ein Unternehmen über Jahrhunderte. Es gereicht der französischen Kolonialarbeit zu hoher Ehre.

\section{L'IRRIGATION DE LA VALLÉE DU NIGER AU SOUDAN FRANÇAIS}

L'un des plus grands fleuves du monde, le Niger, traverse le Soudan Français. Son cours actuel est différent de celui d'autrefois. Il irriguait, il y a quelques siècles, de vastes régions du sud du Sahara, qui étaient fertiles et peuplées. Par un phénomène de capture, le fleuve fut attiré vers l'est et les terres furent desséchées et les habitants durent émigrer.

L'objectif que se proposa le Gouvernement Français fut de transformer le désert en pays riche et d'améliorer les conditions de vie des Soudanais. Il ne s'agissait pas de créer une entreprise du type capitaliste, recherchant le profit financier, mais de constituer un centre de vie rurale doté d'un équipement moderne et permettant à des Africains de s'élever dans la hiérarchie sociale et d'être associés aussi étroitement que possible au developpement de la production.

Pour atteindre cet objectif, le Gouvernement Français créa en 1932 l'Office du Niger. Le service des Etudes Générales prépare les tâches par ses stations spécialisées dans le riz et le coton. Le service des Travaux Neufs aménage des terres, creuse des canaux et livre à l'exploitation des secteurs prêts à être mis en culture, par intermédiaire de colons africains ou en régie directe.

Le coton de l'office du Niger, d'excellente qualité, trouve facilement des acheteurs. Le riz est vendu au Sénégal et au Soudan. Les perspectives d'avenir sont très belles, car il est possible maintenant d'aménager et d'équiper des centaines de milliers d'hectares. Grâce à l'assistance technique de la France, une région désolée du centre de l'Afrique a été transformée en une source de richesses et de vie. Le désert a fait place à une oasis. 